\title{
"H-states": exact solutions for a rotating hollow vortex
}

\author{
D. G. Crowdy ${ }^{1} \dagger$, R. B. Nelson ${ }^{2}$ and V. S. Krishnamurthy ${ }^{3}$ \\ ${ }^{1}$ Department of Mathematics, Imperial College London, London SW7 2AZ, UK \\ ${ }^{2}$ Department of Earth Science and Engineering, Imperial College London, London SW7 2AZ, \\ UK \\ ${ }^{3}$ Faculty of Mathematics, University of Vienna, Oskar-Morgenstern-Platz 1, 1090 Vienna, \\ Austria
}

(Received $\mathrm{xx}$; revised $\mathrm{xx}$; accepted $\mathrm{xx}$ )

Exact solutions are found for an $N$-fold rotationally symmetric, steadily-rotating hollow vortex where a continuous real parameter governs its deformation from a circular shape and $N \geqslant 2$ is an integer. The vortex shape is found as part of the solution. Following the designation "V-states" assigned to steadily rotating vortex patches [Deem \& Zabusky, Phys. Rev. Lett., 40, (1978)] we call the analogous rotating hollow vortices "H-states". Unlike V-states where all but the $N=2$ solution - the Kirchhoff ellipse - must be found numerically, it is shown that all $\mathrm{H}$-state solutions can be written down in closed form. Surface tension is not present on the boundaries of the rotating H-states but the latter are shown to be intimately related to solutions for a non-rotating hollow vortex with surface tension on its boundary [Crowdy, Phys. Fluids, 11, (1999)]. It is also shown how the results here relate to recent work on constant-vorticity water waves [Hur \& Wheeler, J. Fluid Mech., 896, (2020)] where a connection to classical capillary waves [Crapper, J. Fluid Mech., 2, (1957)] is made.

Key words: vortex dynamics, vortex flows, quadrature domains

\section{Introduction}

The two most popular models of a distributed vortex structure - in which vorticity is not concentrated at a single point as is the case for a point vortex - are the vortex patch and the hollow vortex (Saffman 1992). In the vortex patch model vorticity is non-zero and uniform in a bounded region, or patch, of fluid (Newton 2001; Saffman 1992). The circular patch is known as the Rankine vortex and the rotating elliptical patch is the Kirchhoff vortex (Newton 2001; Saffman 1992). Nowadays a rotating vortex patch is commonly given the name "V-state" after an important paper by Deem \& Zabusky (1978) which, arguably, reinvigorated interest in the vortex patch model not only by identifying $N$-fold rotationally symmetric dispersive wave solutions of the two-dimensional Euler equations (the "V-states") but also by indicating how they may be computed numerically using contour dynamics methods which quickly became a popular tool (Pullin 1992). Shortly after the paper by Deem \& Zabusky (1978), Saffman \& Szeto (1980) computed the shapes of a pair of corotating (like-signed) vortex patches. Studying vortex pairs is an important paradigm in understanding basic vortex interactions (Meunier et al. 2002).

$\dagger$ Email address for correspondence: d.crowdy@imperial.ac.uk 


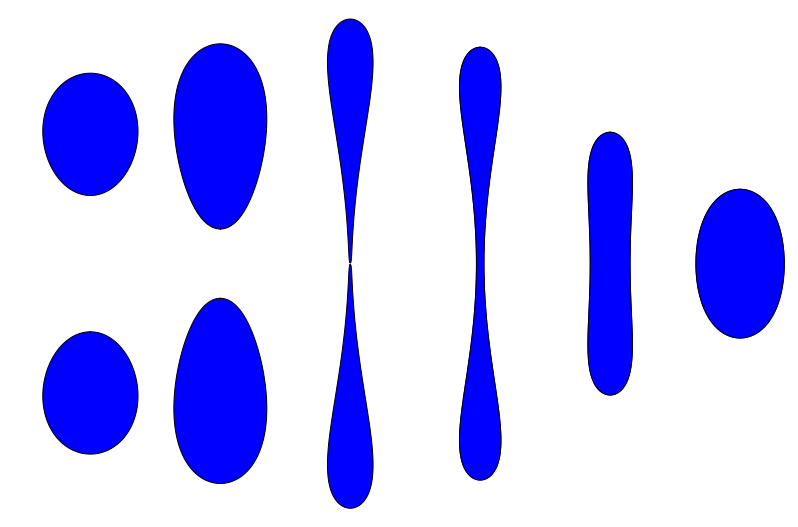

FiguRE 1. Shapes of two steadily-rotating hollow vortices, in the corotating frame, as computed numerically by Nelson et al. (2020). The present paper shows that the single rotating hollow vortices in the three right-most images can in fact be described in analytical form: they are the $N=2$ case of a class of $N$-fold-symmetric "H-states".

A hollow vortex is usually defined to be a finite-area constant pressure region having a non-zero circulation around it (Michell 1890; Baker et al. 1976; Saffman 1992). Although Pocklington (1895) solved the cotravelling (opposite-signed) hollow vortex pair problem in the 19th century, the basic problem of the corotating (like-signed) hollow vortex pair has only recently been treated by Nelson et al. (2020). Indeed there has been a resurgence of interest in the hollow vortex model. One reason is that it is a useful model for incorporating the effects of compressibility (Ardalan et al. 1995; Crowdy \& Krishnamurthy 2017); another is that free streamline theory (Lamb 1994) can be used to find analytical solutions. Pocklington's solution (Pocklington 1895) has been reappraised by Crowdy et al. (2013) using a so-called prime function (Crowdy 2020). Baker et al. (1976) gave an analytical solution for a periodic row of hollow vortices while Crowdy \& Green (2011) found analytical solutions for the hollow-vortex analogue of von Kármán's staggered point vortex street. Analytical solutions for a steady hollow vortex in a linear strain were found by Llewellyn Smith \& Crowdy (2012); this was extended by Zannetti et al. (2016) to hollow vortices in shear (analytical solutions appear not to be available in this case). Crowdy \& Roenby (2014) found exact solutions for a steady hollow vortex surrounded by an $N$-fold polygonal array of point vortices thus generalizing a pointvortex study by Morikawa \& Swenson (1971). Those authors also identified solutions for steadily translating water waves with a cotravelling submerged point vortex row (Crowdy \& Roenby 2014).

When a hollow vortex is steadily rotating an analytical treatment using free streamline theory is not straightforward since, on moving to a corotating frame, uniform vorticity is introduced. Nelson et al. (2020) instead devised a numerical method tailored to account for the doubly connected nature of the fluid exterior to the two vortices. As the angular velocity increases, each vortex is found to extend a thin finger towards the centre of rotation until the vortices almost touch; this sequence is shown from left to right in Figure 1. Nelson et al. (2020) adapted their numerical scheme to compute the shape of a single 2-fold rotationally-symmetric rotating hollow vortex. In that case a thin waist forms in the vortex shape which eventually collapses; this sequence is shown from right to left in Figure 1. Both sequences in Figure 1 are found to approach the same limiting state. Nelson et al. (2020) argue this to be evidence of a topological singularity since the 
same state is approached from two topologically-distinct directions but with no blow-up of any physical quantities.

The purpose of this paper is to point out, first, that 2-fold rotationally-symmetric solutions for a single hollow vortex computed numerically by Nelson et al. (2020) can, in fact, be written down analytically. It is then shown that this solution is just the $N=2$ case of a family of solutions for $N$-fold rotationally-symmetric rotating hollow vortices where $N \geqslant 2$ is an integer. Following Deem \& Zabusky (1978), who used "Vstates" to refer to steadily-rotating vortex patches, the analogous rotating hollow vortices derived here will be called "H-states". For V-states, the $N=2$ solution is the celebrated rotating Kirchhoff ellipse which is a well-known exact solution of the two-dimensional Euler equations (Newton 2001; Saffman 1992); none of the $N>2$ V-states, however, can be written down in closed form and must be computed numerically (Deem \& Zabusky 1978). All H-state solutions, on the other hand, can be written down explicitly for any $N \geqslant 2$ as will be shown.

\section{The H-state problem}

The challenge is to find a single hollow vortex with unchanging shape, an "H-state", with non-zero circulation $\Gamma$ in steady solid body rotation with angular velocity $\Omega$. The interior of the vortex is a constant pressure region. The flow $\mathbf{u}=(u, v)$ is incompressible so to describe the flow exterior to the vortex in this corotating frame we can introduce a streamfunction $\psi(x, y)$ such that

$$
u=\frac{\partial \psi}{\partial y}, \quad v=-\frac{\partial \psi}{\partial x} .
$$

Exterior to the vortex the streamfunction $\psi$ satisfies

$$
\nabla^{2} \psi=-\omega=2 \Omega
$$

The kinematic condition that the vortex boundary is a streamline in the corotating frame, together with Bernoulli's theorem (Batchelor 2000; Saffman 1992) and the condition that the pressure is continuous imply that, on the vortex boundary,

$$
\mathbf{u} \cdot \mathbf{n}=0, \quad \mathbf{u} \cdot \mathbf{t}=q,
$$

where $\mathbf{n}$ is the outward normal to the vortex boundary and $\mathbf{t}$ is the tangent vector as the boundary is traversed in an anticlockwise direction. The constant $q$ is the fluid speed around the boundary. On introducing the complex variable $z=x+\mathrm{i} y$ the two boundary conditions $(2.3)$ can be written in complex form as

$$
u+\mathrm{i} v=q \frac{d z}{d s}
$$

where $d z / d s$ is the complex tangent and $d s$ is the arclength element that increases anticlockwise around the boundary. Equation (2.2) takes the form

$$
\frac{\partial^{2} \psi}{\partial z \partial \bar{z}}=\frac{\Omega}{2}
$$

which allows an integration with respect to $z$ and $\bar{z}$ :

$$
\psi=\frac{\Omega}{2} z \bar{z}+\operatorname{Im}[w(z)]
$$


where the analytic function $w(z)$ is the complex potential for an irrotational flow component exterior to the vortex. From (2.1) and (2.6) it can be deduced that

$$
u-\mathrm{i} v=2 \mathrm{i} \frac{\partial \psi}{\partial z}=\mathrm{i} \Omega \bar{z}+\frac{d w}{d z} .
$$

It follows from (2.4) and (2.7) that, on the H-state boundary,

$$
\mathrm{i} \Omega \bar{z}+\frac{d w}{d z}=q \frac{d \bar{z}}{d s} .
$$

This boundary condition will determine both the H-state shape and the flow around it. The total circulation $\Gamma$ of the H-state is

$$
\Gamma=q \mathcal{P}+2 \Omega \mathcal{A}
$$

comprising a contribution from the constant tangential velocity $q$ around the perimeter $\mathcal{P}$ and from the uniform vorticity $2 \Omega$ over the H-state area $\mathcal{A}$. The relation (2.9) follows on integrating (2.8) with respect to $d z$ around the H-state boundary.

\section{Exact solutions for H-states}

It will be shown that the class of conformal mappings from the interior of the unit disc in a complex $\zeta$-plane to the region exterior to an H-state in the corotating $z$-plane is

$$
z=Z(\zeta)=-\frac{R}{\zeta}\left[1+\frac{4 N}{(N-1)^{2}} \frac{\zeta^{N}}{\zeta^{N}-a^{N}}\right], \quad 1<a_{\text {crit }}^{(N)}<a, \quad N \geqslant 2,
$$

where $R>0$ is a real normalization parameter that sets the size of the H-state. The limit $a \rightarrow \infty$ retrieves the circular hollow vortex of radius $R$. For each $N \geqslant 2$ there is a minimum critical value of $a$, denoted by $a_{\text {crit }}^{(N)}$, below which the shapes described by (3.1) are not univalent and are therefore not physically admissible; this loss of univalency is brought about by two distinct parts of the vortex boundary coming into contact at $a=a_{\text {crit }}^{(N)}$ as will we seen later in Figure 2. Actually, the class of conformal mappings (3.1) was first written down by Crowdy (1999a) for $N=2$, and for $N>2$ (in a modified but equivalent form using the exterior of the unit disc as the preimage domain) by Wegmann \& Crowdy (2000). The same mappings (3.1) are also used by Crowdy \& Roenby (2014). All this will be discussed in $\S 4$. Crowdy $(1999 a)$ reports $a_{\text {crit }}^{(2)}=3.000$. The values $a_{\text {crit }}^{(3)}=1.690, a_{\text {crit }}^{(4)}=1.400, a_{\text {crit }}^{(5)}=1.277$ can be derived using the formula $a_{\text {crit }}^{(N)}=$ $\left[(N-1)^{1 / N} \tilde{a}_{\text {crit }}^{(N)}\right]^{-1}$ where $\tilde{a}_{\text {crit }}^{(N)}$ are the critical values of Wegmann \& Crowdy (2000) who found numerically that $\tilde{a}_{\text {crit }}^{(3)}=0.4696, \tilde{a}_{\text {crit }}^{(4)}=0.5426$ and $\tilde{a}_{\text {crit }}^{(5)}=0.5934$. Formula (3.1) implies that on the H-state boundary, or $|\zeta|=1$,

$$
\bar{Z}\left(\zeta^{-1}\right)=-R \zeta\left[1+\frac{4 N}{(N-1)^{2}} \frac{1}{1-\zeta^{N} a^{N}}\right],
$$

where $\bar{Z}(\zeta)=\overline{Z(\bar{\zeta})}$ denotes the Schwarz conjugate function. It can also be verified, by direct differentiation, that

$$
\begin{aligned}
\zeta Z^{\prime}(\zeta) & =\frac{R}{(N-1)^{2} \zeta}\left[\frac{(N+1) \zeta^{N}+(N-1) a^{N}}{\left(\zeta^{N}-a^{N}\right)}\right]^{2}, \quad Z^{\prime}(\zeta) \equiv \frac{d Z}{d \zeta}, \\
\zeta^{-1} \bar{Z}^{\prime}\left(\zeta^{-1}\right) & =\frac{R \zeta}{(N-1)^{2}}\left[\frac{(N+1)+(N-1) \zeta^{N} a^{N}}{\left(1-\zeta^{N} a^{N}\right)}\right]^{2},
\end{aligned}
$$


and consequently that

$$
\frac{\zeta^{-1} \bar{Z}^{\prime}\left(\zeta^{-1}\right)}{\zeta Z^{\prime}(\zeta)}=\zeta^{2}\left[\frac{(N+1)+(N-1) \zeta^{N} a^{N}}{\left(1-\zeta^{N} a^{N}\right)} \times \frac{\left(\zeta^{N}-a^{N}\right)}{(N+1) \zeta^{N}+(N-1) a^{N}}\right]^{2} .
$$

Note from (3.3) the interesting features that $d Z / d \zeta$ is a square of a rational function of $\zeta$, and has a rational function primitive. The boundary condition $(2.8)$ valid on $|\zeta|=1$ implies that, on the H-state boundary,

$$
\frac{d w}{d z}=q \frac{d \bar{z}}{d s}-\mathrm{i} \Omega \bar{z}=\mathrm{i} q\left[\frac{\zeta^{-1} \bar{Z}^{\prime}\left(\zeta^{-1}\right)}{\zeta Z^{\prime}(\zeta)}\right]^{1 / 2}-\mathrm{i} \Omega \bar{Z}(1 / \zeta),
$$

where we have used the fact that $\bar{\zeta}=1 / \zeta$ on this boundary. On use of (3.2) and (3.4),

$$
\begin{aligned}
& \frac{d w}{d z}=\mathrm{i} q \zeta {\left[\frac{(N+1)+(N-1) \zeta^{N} a^{N}}{\left(1-\zeta^{N} a^{N}\right)} \times \frac{\left(\zeta^{N}-a^{N}\right)}{(N+1) \zeta^{N}+(N-1) a^{N}}\right] } \\
&+\mathrm{i} \Omega R \zeta\left[1+\frac{4 N}{(N-1)^{2}} \frac{1}{1-\zeta^{N} a^{N}}\right], \quad \text { on }|\zeta|=1,
\end{aligned}
$$

where we can think of the right hand side as a function of $z$ using the inverse function, $\zeta=Z^{-1}(z)$, of the conformal mapping (3.1). Both sides of (3.6) are analytic functions of $z$ that can be continued off the H-state boundary into the fluid domain exterior to it, or equivalently, inside the unit $\zeta$-disc. Remarkably, the right hand side is a rational function of $\zeta$. Since we require $d w / d z$ to be free of singularities in the fluid region, and hence in $|\zeta|<1$, it is necessary to remove the $N$ simple poles of the right hand side of (3.6) at the roots of $\zeta^{N}=1 / a^{N}$ which are inside the unit $\zeta$-disc because $a>1$. This can be done, by virtue of the $N$-fold rotational symmetry, by the single condition

$$
q=\frac{2 \Omega R}{(N-1)^{2}}\left[\frac{(N-1) a^{2 N}+N+1}{a^{2 N}-1}\right]
$$

obtained by setting the coefficient of $\left(1-\zeta^{N} a^{N}\right)^{-1}$ evaluated at $\zeta=1 / a$ on the right hand side of (3.6) equal to zero. Since, for the univalency of the mapping, $d Z / d \zeta$ must not vanish inside the unit disc, the right hand side of (3.6) has no other singularities in this disc and therefore $d w / d z$ is analytic there, as required. Since as $\zeta \rightarrow 0$, or equivalently as $z \rightarrow \infty$, it follows from (3.6) and (3.1) that

$$
\frac{d w}{d z} \sim-\frac{\mathrm{i} R}{z}\left[-q\left(\frac{N+1}{N-1}\right)+\Omega R\left(1+\frac{4 N}{(N-1)^{2}}\right)\right]
$$

and since it is required that

$$
\frac{d w}{d z} \sim-\frac{\mathrm{i} \Gamma}{2 \pi z}, \quad \text { as } z \rightarrow \infty,
$$

then a comparison of (3.8) and (3.9) shows it necessary to pick parameters satisfying

$$
\frac{\Gamma}{2 \pi R}=-q\left(\frac{N+1}{N-1}\right)+\Omega R\left(1+\frac{4 N}{(N-1)^{2}}\right) .
$$

Substitution of condition (3.7) into (3.10) produces

$$
\Omega=\frac{\Gamma}{2 \pi R^{2}} /\left[1+\frac{4 N}{(N-1)^{2}}-\frac{2(N+1)}{(N-1)^{3}}\left(\frac{(N-1) a^{2 N}+N+1}{a^{2 N}-1}\right)\right]
$$

which, for a given value of $\Gamma$, is an explicit expression for the angular velocity $\Omega$ in terms of the geometrical parameters $R, a$ and $N$. With $\Omega$ thus determined, (3.7) gives $q$. With 
all parameters now known the velocity field follows, as an explicit function of $\zeta$, from (2.7) and the analytic continuation of (3.6) as

$$
\begin{aligned}
u-\mathrm{i} v=\mathrm{i} \Omega \overline{Z(\zeta)} & +\mathrm{i} q \zeta\left[\frac{(N+1)+(N-1) \zeta^{N} a^{N}}{\left(1-\zeta^{N} a^{N}\right)} \times \frac{\left(\zeta^{N}-a^{N}\right)}{(N+1) \zeta^{N}+(N-1) a^{N}}\right] \\
& +\mathrm{i} \Omega R \zeta\left[1+\frac{4 N}{(N-1)^{2}} \frac{1}{1-\zeta^{N} a^{N}}\right] .
\end{aligned}
$$

The solution is complete and the H-states have been determined parametrically as explicit functions of $\zeta$ for any $N \geqslant 2$. Conveniently, from the integral expressions

$$
\mathcal{P}=\oint_{|\zeta|=1}\left|\frac{d Z}{d \zeta}\right| \frac{d \zeta}{\mathrm{i} \zeta}, \quad \mathcal{A}=-\frac{1}{2 \mathrm{i}} \oint_{|\zeta|=1} \bar{Z}\left(\zeta^{-1}\right) \frac{d Z}{d \zeta} d \zeta
$$

and use of residue calculus, it is easy to show that

$$
\begin{aligned}
& \mathcal{P}=2 \pi R\left[\frac{2 N}{(N-1)^{2}}\left(\frac{(N-1) a^{2 N}+N+1}{a^{2 N}-1}\right)-\frac{N+1}{N-1}\right] \\
& \mathcal{A}=\frac{\pi R^{2}}{\left(1-a^{2 N}\right)^{2}(N-1)^{2}}\left[\left(\frac{N+1}{N-1}\right)^{2}\left(N^{2}-6 N+1\right)-2\left(\frac{N+1}{N-1}\right)\left(N^{2}+4 N-1\right) a^{2 N}\right. \\
&\left.+a^{4 N}(N-1)^{2}\right] .
\end{aligned}
$$

Such formulas are useful since if one seeks H-states with fixed area $\mathcal{A}=\pi$, say, then the second formula in (3.14) gives an explicit expression for $R$ in terms of $N$ and $a$. With parameters $q, \Omega, \mathcal{P}$ and $\mathcal{A}$ determined by (3.7), (3.11) and (3.14), a consistency check on the solution is provided by confirming that (2.9) holds.

A purely numerical method to compute the H-state for $N=2$ was given in Nelson et al. (2020) where the values of $q$ and $\Omega$ associated with the critical state, corresponding to $a=a_{\text {crit }}^{(2)}=3.000$, are reported for $\Gamma=2$ and $\mathcal{A} \approx 2 \times 0.311$. As a check on the above analysis, we can compute $q$ and $\Omega$ using (3.7) and (3.11) with $\Gamma=2$, and $R$ calculated from the second formula in (3.14) with $\mathcal{A}=\pi$, to find $q \approx 0.236$ and $4 \pi \Omega \approx 1.347$ which coincide, to within numerical accuracy, to the (suitably rescaled) values reported by Nelson et al. (2020). A comparison of the shapes given by formula (3.1) for $N=2$ with those calculated numerically by Nelson et al. (2020) reveals them to be indistinguishable for arbitrary parameter values.

H-state shapes are shown for $N=2,3,4$ and 5 in Figure 2. At $a=a_{\text {crit }}^{(N)}$ the H-states show an $N$-fold rotationally symmetric pinch-off where different parts of the boundary come into contact. These are quite different to the critical V-state shapes which are known to exhibit $90^{\circ}$ corner formation (Overman 1986; Saffman 1992).

Figure 3 shows graphs of $\Omega$ and $q$ against $a$ for $N=2,3,4$ and 5 for $\Gamma=1$ and $\mathcal{A}=\pi$. A bifurcation analysis from the circular state - that is, setting $Z(\zeta)=-\zeta^{-1}+\epsilon \zeta^{N-1}$ in (3.5) and expanding for small $\epsilon \ll 1$ with $\Gamma=1$ - leads to

$$
\Omega \sim \frac{N-1}{2 \pi(N+1)}, \quad q \sim \frac{1}{\pi(N+1)} .
$$

The curves in Figure 3 tend to the values in (3.15) as $a \rightarrow \infty$ which corresponds to the near-circular state. As $a$ decreases from infinity $\Omega$ and $q$ remain close to the values (3.15) until $a$ gets close to $a_{\text {crit }}^{(N)}$ when they typically decrease monotonically (although a small increase in $q$ for $N=5$ is observed before it decreases). 

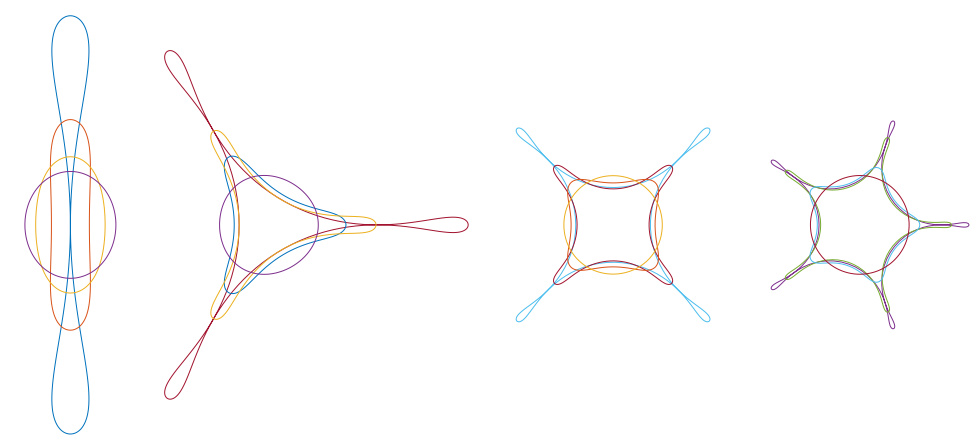

Figure 2. H-states for $\mathcal{A}=\pi$ and (from left to right) $N=2,3,4$ and 5. Parameter values are: $N=2, a=a_{\text {crit }}^{(2)}(=3.000), 3.5,5,10 ; N=3, a=a_{\text {crit }}^{(3)}(=1.690), 1.8,2,5$; $N=4, a=a_{\text {crit }}^{(4)}(=1.400), 1.5,1.8,5 ; N=5, a=a_{\text {crit }}^{(5)}(=1.277), 1.3,1.5,5$.
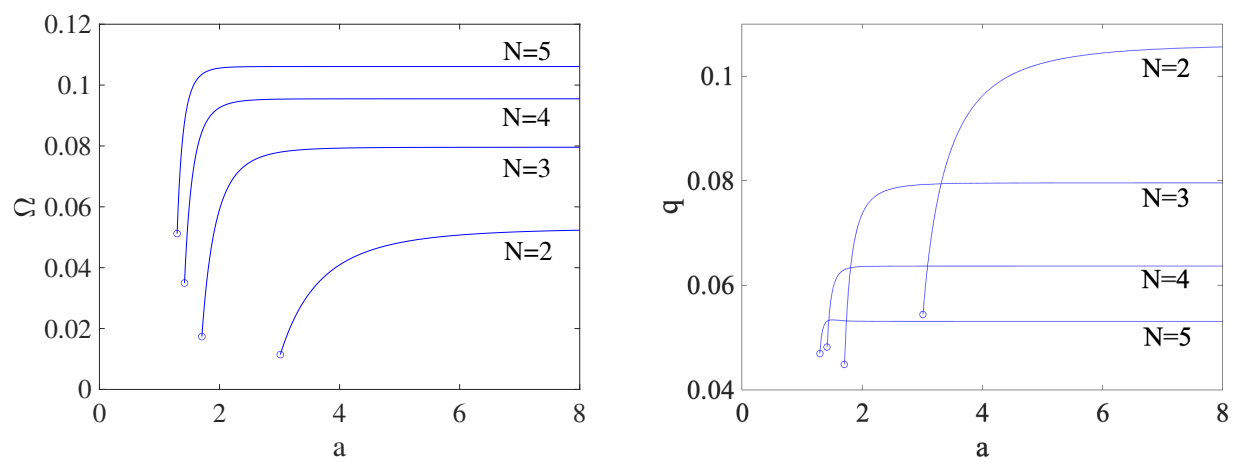

Figure 3. Graphs of (left) $\Omega$ and (right) $q$ against $a$ for $N=2,3,4$ and 5 and $\Gamma=1, \mathcal{A}=\pi$.

\section{Perspectives}

It is surprising that these exact solutions for such a basic class of distributed vortex structures have escaped notice for so long. This is likely due to the aforementioned observation that classical free streamline theory is unavailable as a route to their derivation. Like the Rankine vortex and the Kirchhoff ellipse, the results are valuable pedagogically in providing mathematically explicit desingularizations of an isolated point vortex.

The class of conformal mappings (3.1) was used first by Crowdy (1999a) and Wegmann \& Crowdy (2000) to find solutions for a non-rotating hollow vortex but with the important difference that surface tension acts on its boundary. The boundary conditions are then more complicated because the fluid pressure is no longer constant on the vortex boundary but is balanced by a curvature-dependent surface tension term. As has been shown here, the class of shapes solving that quite different free boundary problem coincide with the H-state shapes found here. Indeed there is yet another distinct free boundary problem also solved by the same class of mappings (3.1): Crowdy \& Roenby (2014) found that they also solve the free boundary problem of a central hollow vortex, without surface tension, in equilibrium with an $N$-polygonal array of satellite point vortices.

The work of Crowdy (1999a) and Wegmann \& Crowdy (2000) emerged from insights gained from a new approach to free surface Euler flows with surface tension propounded 
by Crowdy (2000). There the author proposed a conformal mapping approach to understand why the classic problem of pure capillary waves on deep water found by Crapper (1957) admits exact solutions; he also sought to understand how Crapper's solution relates to analytical observations made by Tanveer (1996) on the functional form of conformal mappings to the region exterior to a translating bubble with surface tension, another example of a free surface Euler flow with surface tension. Crapper (1957) used hodograph variables but it is not clear from his approach why his proposed solution ansatz works. Crowdy (2000) gained more insight by observing a Riccati-type structure to the analytically-continued boundary condition that allowed deductions to be made on the functional form of the conformal mapping function. He showed why Crapper's $2 \pi$-periodic solution must be given by the following log-rational mapping, from the unit $\eta$-disc, to a physical $\mathcal{Z}$ plane

$$
\mathcal{Z}=\tilde{Z}(\eta)=\mathrm{i}\left[\log \eta-\frac{4 \hat{a}}{\eta-\hat{a}}\right], \quad \hat{a}>1 .
$$

The arguments of Crowdy (2000) can be adapted to the present H-state problem to argue why the relevant conformal mappings must have the functional form (3.1).

In view of these analytical connections (Crowdy 2000) between Crapper's capillary waves and hollow vortices with surface tension (Crowdy 1999a; Wegmann \& Crowdy 2000), and since the same mappings (3.1) used in the latter problem also solve the H-state problem, it is natural to ask if the new H-state results in this "radial geometry" might produce analogous exact solutions to some problem in Crapper's periodic water wave geometry. It turns out that such solutions have very recently been discovered by Hur \& Wheeler (2020) whose work was motivated by a string of other recent contributions (Hur \& Vanden-Broeck 2020; Hur \& Dyachenko 2019a,b) where it was noticed that Crapper's capillary wave profiles were emerging in numerical simulations of rotational water waves. A similar thing happened here: noticing that the critical shape from Nelson et al. (2020) as shown in Figure 1 is indistinguishable from the critical shape shown in Figure 1 of Crowdy $(1999 a)$ led to the new H-state solutions. On making the identifications

$$
\mathcal{Z}=-\mathrm{i} \log z^{N}, \quad \eta=\zeta^{N}, \quad \hat{a}=a^{N}
$$

with $z$ related to $\zeta$ via (3.1) it can be shown that (4.1) is retrieved (to within unimportant additive constants) as $N \rightarrow \infty$. That is, the $N \rightarrow \infty$ limit of the H-states reproduces the water wave solutions of Hur \& Wheeler (2020).

Actually, the surprising reappearance of Crapper's profiles in a problem different from the original problem of capillary water waves was noticed earlier by Crowdy \& Roenby (2014) who found exact solutions, given by Crapper's profiles (4.1), for steady water waves with vorticity: in their problem, the vorticity in each period window is concentrated in a submerged cotravelling point vortex. In view of the recent results of Hur \& Wheeler (2020) the potential theoretic concept of "balayage" (Shapiro 1992) comes to mind: one imagines that the uniform vorticity in each period window in the Hur \& Wheeler (2020) solutions is "swept" into a single point vortex to give the solutions of Crowdy \& Roenby (2014) and without changing the wave profile.

It is intriguing that the same classes of mapping functions - the radial geometry shapes embodied in (3.1) and the Crapper-type periodic waves encoded in (4.1) - appear to be "canonical" in that they recur in at least three physically distinct problems. As discussed by Crowdy \& Roenby (2014), this is likely due to the fact that (3.1) and (4.1) are the conformal mappings to so-called double quadrature domains which form an important class having their own mathematical significance; this is related to the features, noted in $\S 3$, that $d Z / d \zeta$ is both a square of a rational function of $\zeta$ and has a rational function 
primitive. A recent monograph (Crowdy 2020) makes the case that the class of quadrature domains should be known more widely in the applied and physical sciences. They occur in various guises in fluid dynamics as surveyed by Crowdy (2005). We mention here that other classes of exact solutions - also viewable as quadrature domains - for steadilytranslating water waves with vorticity have been found by Crowdy \& Nelson (2010).

The results here point to the intriguing possibility that other analytical solutions exist for rotating hollow vortex equilibria or those with background vorticity. In the corotating frame the H-states have a combination of uniform vorticity and concentrated boundary vorticity. They have these features in common with Sadovskii vortices (Saffman 1992) which are vortex structures that have also received renewed attention (Freilich \& Llewellyn Smith 2017). Zannetti et al. (2016) computed the steady shapes of a hollow vortex in simple shear numerically, but it is conceivable that the analytical observations here are extendible to that problem which has many elements in common. Of course, whether the corotating hollow vortex pair calculated numerically by Nelson et al. (2020) - i.e. the detached vortex pairs shown on the left in Figure 1 - also admits analytical solutions is an open problem. In this regard it should be mentioned that, using the prime function for a concentric annulus (Crowdy 2020), the author has extended the work of Crowdy (1999a) and Wegmann \& Crowdy (2000) to find exact solutions for capillary waves on a fluid annulus with two free surfaces (Crowdy 1999b, 2001). Crowdy (1999b) also offered alternative forms, generalizing (4.1), of the capillary wave solutions on fluid sheets found by Kinnersley (1977) who used Jacobi elliptic functions to generalize Crapper's solution. A similar approach using the prime function (Crowdy et al. 2013) also obviated the need for Jacobi elliptic functions in describing Pocklington's cotravelling hollow vortex pair (Pocklington 1895) and facilitated the calculation of its linear stability properties. The novel prime function approach to Kinnersley's solutions in Crowdy $(1999 b)$ might similarly uncover new solutions for water waves with vorticity on fluid sheets thereby generalizing the results of Hur \& Wheeler (2020).

The stability of the H-states is clearly of interest, and can be studied using techniques similar to those used by Llewellyn Smith \& Crowdy (2012) and Crowdy et al. (2013). This matter remains to be investigated.

\section{REFERENCES}

Ardalan, K., Meiron, D. I. \& Pullin, D. I. 1995 Steady compressible vortex flows: the hollow-core vortex array. J. Fluid Mech. 301, 1.

Baker, G. R., Saffman, P. G. \& Sheffield, J. S. 1976 Structure of a linear array of hollow vortices of finite cross-section. J. Fluid Mech. 74, 469-476.

Batchelor, G. K. 2000 An Introduction to Fluid Dynamics. Cambridge University Press.

CrApper, G. 1957 An exact solution for progressive capillary waves of arbitrary amplitude. $J$. Fluid Mech. 2, 532-540.

Crowdy, D. G. 1999 a Circulation-induced shape deformations of drops and bubbles: exact two-dimensional models. Phys. Fluids 11, 2836-2845.

Crowdy, D. G. $1999 b$ Exact solutions for steady capillary waves on a fluid annulus. J. Nonlin. Sci. 9, 615-640.

Crowdy, D. G. 2000 A new approach to free surface Euler flows with capillarity. Stud. Appl. Math. 105, 35-58.

Crowdy, D. G. 2001 Steady nonlinear capillary waves on curved sheets. Eur. J. Appl. Math. 12, 689-708.

Crowdy, D. G. 2005 Quadrature domains and fluid dynamics. In Quadrature domains and applications (ed. Khavinson-Putinar Ebenfelt, Gustafsson). Birkhauser.

Crowdy, D. G. 2020 Solving problems in multiply connected domains. Society for Industrial and Applied Mathematics. 
Crowdy, D G \& Green, C C 2011 Analytical solutions for von Kármán streets of hollow vortices. Phys. Fluids 23, 126602.

Crowdy, D. G. \& Krishnamurthy, V. S. 2017 The effect of core size on the speed of compressible hollow vortex streets. J. Fluid Mech. 836, 797-827.

Crowdy, D. G., Llewellyn Smith, S. G. \& Freilich, D. V. 2013 Translating hollow vortex pairs. European Journal of Mechanics B/Fluids 37, 180-186.

Crowdy, D. G. \& Nelson, R. B. 2010 Steady interaction of a vortex street with a shear flow. Phys. Fluids 22, 096601.

Crowdy, D. G. \& Roenby, J. 2014 Hollow vortices, capillary water waves and double quadrature domains. Fluid Dyn. Res. 46, 031424.

Deem, G S \& Zabusky, N J 1978 Vortex waves: Stationary 'V-states', interactions, recurrence and breaking. Phys. Rev. Lett. 40, 859-862.

Freilich, D. V. \& Llewellyn Smith, S. G. 2017 The Sadovskii vortex in strain. J. Fluid Mech. 825, 479-501.

Hur, V. M. \& Dyachenko, S. A. $2019 a$ Stokes waves with constant vorticity: folds, gaps and fluid bubbles. J. Fluid Mech. 878, 502-521.

Hur, V. M. \& Dyachenko, S. A. $2019 b$ Stokes waves with constant vorticity: I. Numerical computation. Stud. Appl. Math. 142, 162-189.

Hur, V. M. \& Vanden-Broeck, J.-M. 2020 A new application of Crapper's exact solution to waves in constant vorticity flows. Eur. J. Mech. (B/Fluids) 83, 190-194.

Hur, V. M. \& Wheeler, M. H. 2020 Exact free surfaces in constant vorticity flows. J. Fluid Mech. 896, R1.

Kinnersley, W. 1977 Exact large amplitude capillary waves on sheets of fluid. J. Fluid Mech. 77, 229-241.

Lamb, H. 1994 Hydrodynamics. Cambridge University Press.

Llewellyn Smith, S. G. \& Crowdy, D. G. 2012 Structure and stability of hollow vortex equilibria. J. Fluid Mech. 691, 178-200.

Meunier, P., Ehrenstein, U., Leweke, T. \& Rossi, M. 2002 A merging criterion for twodimensional co-rotating vortices. Phys. Fluids 14 (8), 2757-2766.

Michell, J. H. 1890 On the theory of free stream lines. Phil. Trans. Roy. Soc. Lond 181, 389-431.

Morikawa, G. K. \& Swenson, E. V. 1971 Interacting motion of rectilinear geostrophic vortices. Phys. Fluids 14, 1058.

Nelson, R. B., Krishnamurthy, V. S. \& Crowdy, D. G. 2020 The corotating hollow vortex pair: steady merger and break-up via a topological singularity. J. Fluid Mech. 907, A10.

Newton, P. K. 2001 The N-Vortex Problem. Analytical Techniques. New York: Springer.

Overman, E. A. 1986 Steady-state solutions of the Euler equations in two dimensions II: local analysis of limiting V-states. SIAM J. Appl. Math. 46, 765-800.

Pocklington, H. C. 1895 The configuration of a pair of equal and opposite hollow straight vortices of finite cross-section, moving steadily through fluid. Proc. Cambridge Philos. Soc. 8, 178-187.

Pullin, D. I. 1992 Contour dynamics methods. Ann. Rev. Fluid Mech. 24, 89-115.

Saffman, P. G. 1992 Vortex Dynamics. Cambridge University Press, Cambridge.

Saffman, P. G. \& Szeto, R 1980 Equilibrium shapes of a pair of equal uniform vortices. Phys. Fluids 23, 2339.

ShaPIRO, H. S. 1992 The Schwarz function and its generalization to higher dimensions. John Wiley \& Sons.

TAnveer, S. 1996 Some analytical properties of solutions to a two-dimensional steadily translating inviscid bubble. Proc. Roy. Soc. A 452, 1397-1410.

Wegmann, R. \& Crowdy, D. G. 2000 Shapes of two-dimensional bubbles deformed by circulation. Nonlinearity 13, 2131-2141.

Zannetti, L., Ferlauto, M. \& Llewellyn Smith, S. G. 2016 Hollow vortices in shear. J. Fluid Mech. 809, 705-715. 